\title{
Bridge Continuous Deformation Measurement Technology Based on Fiber Optic Gyro
}

\author{
Weibing $\mathrm{GAN}^{{ }^{*}}$, Wenbin $\mathrm{HU}^{2}$, Fang $\mathrm{LIU}^{2}$, Jianguang TANG ${ }^{2}$, \\ Sheng $\mathrm{LI}^{2}$, and $\mathrm{Yan} \mathrm{YANG}^{1}$
}

\author{
${ }^{1}$ Key Laboratory of Fiber Optic Sensing Technology and Information Processing of Ministry of Education, Wuhan \\ University of Technology, Wuhan, 430070, China; \\ ${ }^{2}$ National Engineering Laboratory for Fiber Optic Sensing Technology, Wuhan University of Technology, Wuhan, \\ 430070, China \\ ${ }^{*}$ Corresponding author: Weibing GAN_Ｅmail: ganweibing@whut.edu.cn; gwb_cxd@163.com
}

\begin{abstract}
Bridge is an important part of modern transportation systems and deformation is a key index for bridge's safety evaluation. To achieve the long span bridge curve measurement rapidly and timely and accurately locate the bridge maximum deformation, the continuous deformation measurement system (CDMS) based on inertial platform is presented and validated in this paper. Firstly, based on various bridge deformation measurement methods, the method of deformation measurement based on the fiber optic gyro (FOG) is introduced. Secondly, the basic measurement principle based on FOG is presented and the continuous curve trajectory is derived by the formula. Then the measurement accuracy is analyzed in theory and the relevant factors are presented to ensure the measurement accuracy. Finally, the deformation measurement experiments are conducted on a bridge across the Yangtze River. Experimental results show that the presented deformation measurement method is feasible, practical, and reliable; the system can accurately and quickly locate the maximum deformation and has extensive and broad application prospects.
\end{abstract}

Keywords: Long span bridge; continuous deformation measurement; FOG; structural safety

Citation: Weibing GAN, Wenbin HU, Fang LIU, Jianguang TANG, Sheng LI, and Yan YANG, "Bridge Continuous Deformation Measurement Technology Based on Fiber Optic Gyro," Photonic Sensors, 2016, 6(1): 71-77.

\section{Introduction}

Bridge is a very important part of modern transportation industry, and the safety monitoring is quite necessary for the bridge structure. As science and technology develops, an increasing number of devices are used in bridge monitoring. By analyzing these collected data, people can get knowledge of working conditions of bridges not only in the past, but also in the future. During this process, continuous deformation is essential. Deformation is the key technical parameter which judges the vertical stiffness, load-bearing capacity, and integrality of the bridge. The structure of bridge will deform under the action of the external force, and various diseases such as cracks and pre-stress losses will also lead to the bridge deformation, so the deformation is one of the most important indexes for evaluating the health status of bridge structure [1].

In recent years, the number of methods of

Received: 24 August 2015 / Revised: 18 September 2015

(C) The Author(s) 2015. This article is published with open access at Springerlink.com

DOI: 10.1007/s13320-015-0276-6

Article type: Regular 
measuring bridge deformation has been increasing. For example, the level gauge can only measure the deformation of discrete points, and the measurement process is more complex. Another method of measuring the deformation is to take advantages of the photoelectric level of communicating tubes. Although it has high precision, it is not easy to implement because the needed objective cursor is difficultly decided in the practical measurement. The third method is using the laser measurement system and photoelectric image measurement system, which capture the location of the spot or imaging changes to get the changes of relative position through the optical system, and this method must be fixed on the bridge with the equipment as a reference point. When the device is moved, the system can not get the initial measurement datum and meet the long-term measurement request, and different weather conditions have an effect on the measurement. The fourth method is the global position system (GPS), in which the precision is the rate of "cm". It can monitor the object in the storm and realize the automatic monitoring of three dimensional (3D) coordinates. However, the system is expensive, and it can not be applied in large scale. In summary, the traditional measurement methods have the characteristics of long detection period, high implementation cost, and discrete measurement points, which are easy to omit the potential disease parts of the structure, and it is difficult to meet the requirements of large engineering structures for the rapid, continuous, and accurate deformation measurement [2-9].

In this paper, according to the characteristics of the fiber optic gyro (FOG) sensitive angular velocity, the basic principle and method of FOG for measuring deformation are derived. The continuous deformation measurement system (CDMS) based on the inertial platform is designed, and it is applied to the scale bridge model in laboratory and the actual load of a large bridge.

\section{Principle of measurement}

\subsection{Principle}

Considering the FOG can accurately measure the attitude of the carrier motion, the CDMS can push out the moving track of the carrier by collecting the information of the rotational angular velocity and the linear acceleration of the relative inertia space of the gyroscope and accelerometer. The basic principle is described as shown in Fig. 1.

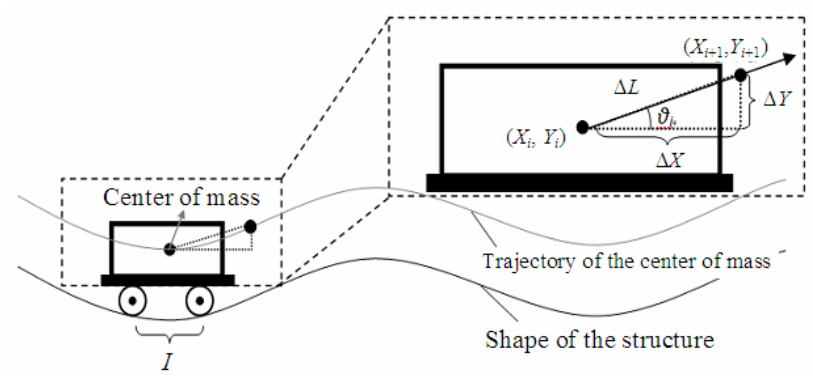

Fig. 1 Principle of CDMS based on FOG.

Here assume that the carrier moves from one point $\left(X_{i}, Y_{i}\right)$ to another point $\left(X_{i+1}, Y_{i+1}\right)$. According to the similarity theory of the integral limit, when the time interval $t$ is very small, the coordinates of point $\left(X_{i+1}, Y_{i+1}\right)$ can be approximated as [10]:

$$
\begin{aligned}
& \left\{\begin{aligned}
X_{i+1} & =X_{i}+\Delta X=X_{i}+\Delta L \cdot \cos \theta_{i+1} \\
& =X_{i}+\Delta L \cdot \cos \left(\theta_{i}+\Delta \theta\right) \\
Y_{i+1} & =Y_{i}+\Delta Y=Y_{i}+\Delta L \cdot \sin \theta_{i+1} \\
& =Y_{i}+\Delta L \cdot \sin \left(\theta_{i}+\Delta \theta\right)
\end{aligned}\right. \\
& \Delta \theta=\int_{t_{i}}^{t_{i}+1} \omega_{i+1} d t, \Delta L=\int_{t_{i}}^{t_{i}+1} v_{i+1} d t .
\end{aligned}
$$

Among them, $V_{i+1}$ is the line speed of the carrier and $\Delta L$ represents the minimum pulse interval. $\omega_{i}$ is the angular velocity, $\Delta \theta$ represents the angle change, and $\theta_{i}$ is the initial angle.

The CDMS algorithm assumes that the length of the carrier, $L$ (shown in Fig. 1), is negligible in comparison with the fluctuations of the detected curve. The calculated trajectory, which is actually the trajectory of the system's center of mass, is regarded as the curve of the object. According to the established formulae $(1,2)$, the system can carry out the continuous curve trajectory calculation. We can get the continuous deformation curve of the bridge by comparing with the design data. 


\subsection{Constitution of the system}

The CDMS consists of five parts, including the rigid four-wheel carrier, data acquisition system, mileage meter and interface circuit, storage battery, and fiber optic inertial navigation system (INS) as shown in Fig. 2.

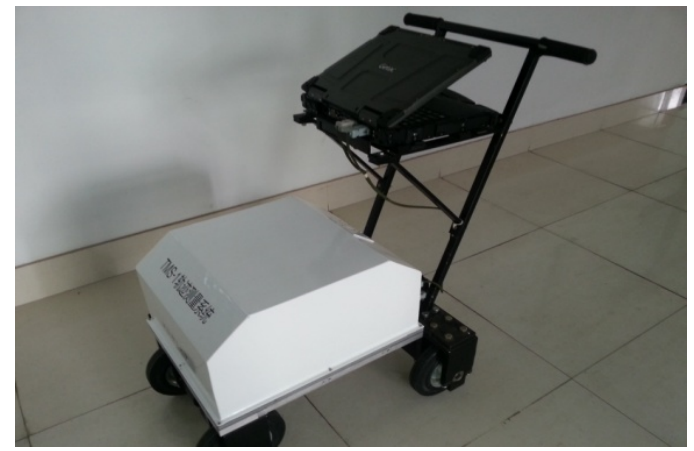

Fig. 2 CDMS based on FOG.

All devices are integrated in the rigid fourwheel carrier, and FOG completes the measurement of angular velocity when the carrier is moving. The mileage meter records the carrier's mileage in real time. In order to ease the bumps caused by uneven pavement, a shock absorber is mounted between the vehicle body and the wheel. The data acquisition system is used to complete the collection of all the devices, and the computer can finish the functions such as receiving data, analyzing the data, and displaying curves in real time by the serial port. By deducing the output information of the fiber optic INS and the mileage meter, we can get the curve trajectory of the carrier running. At the same time, the error of attitude angle and the error of mileage meter are compensated with the position information of the starting point, thus the measurement accuracy can be ensured.

\subsection{Analysis of measurement accuracy}

In this paper, the feasibility of FOG for measuring deformation of engineering structureis is discussed with a cable stayed bridge as an example. The distance between the two spans is $D$, and the height of the chord is $\delta$. The curvature radius of the bridge is $R$, and the angle is $\theta$, as shown in Fig. 3 .

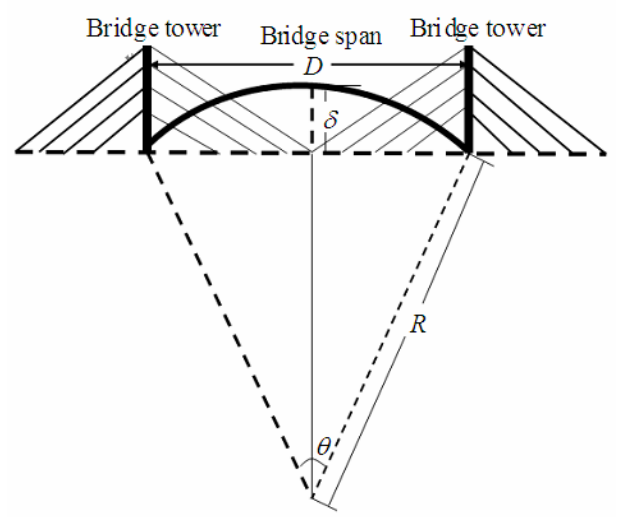

Fig. 3 Structure of cable stayed bridge.

When $R$ is far greater than $D$, the minimum deformation which can be detected by FOG in theory at a given speed can be described by (3):

$$
\delta_{\min }=\Omega_{\min } \cdot D^{2} / 8 V_{\max } .
$$

Among them, $\Omega_{\min }$ is the zero deviation of FOG, and $V_{\max }$ is the maximum line speed of the detection carrier. $\delta_{\text {min }}$ represents the minimum deformation detected by FOG.

The selected FOG is a TXD6-A2 for a company in China, whose zero bias stability is $0.1 \% \mathrm{~h}$, and $\Omega_{\min }$ is $4.845 * 10^{-7} \mathrm{rad} / \mathrm{s}$. Assuming that the maximum speed of the carrier is $5 \mathrm{~km} / \mathrm{h}$ and $D$ is $500 \mathrm{~m}$, then the minimum deformation value can be deduced by (3), which is $0.182 \mathrm{~mm}$. The deformation value can satisfy the accuracy of the deformation measurement for the bridge structure.

\subsection{Factors of ensuring measurement accuracy}

Compared with the long-distance trajectory in the inertial navigation field, the CDMS has the following particularity in the detection process.

(a) The distance is short, and the test can be repeated many times.

(b) The time is short, and the system can neglect the influence of time drift and temperature shift.

(c) The size of the bridge structure is known, and the curve data can be corrected by boundary conditions.

As the FOG is very important in the measurement system, we should analyze some important parameters of the FOG, e.g. the maximum 
input angular rate, temperature effect, scale factor nonlinearity, and random walk coefficient. We just take a few minutes to detect a bridge, so the system can ignore the impact of time and temperature changes. In the process of carrier running, the angular velocity of FOG is between $1 \%$ and $5 \%$, and the nonlinearity of scale factor is about $100 \mathrm{ppm}$, which has little influence on the deformation measurement of bridge. At the same time, considering the systematic error and the random walk of the gyro, the system has adopted the method of initial alignment and the calibration of the known points to ensure the accuracy of the system.

\section{Test of bridge model}

\subsection{Test scenario}

The bridge is a scale model of a Yangtze River Bridge, and the scale is $1: 40$. The length of the main span is $9.7 \mathrm{~m}$, with a height of $3.46 \mathrm{~m}$ and a width of $0.55 \mathrm{~m}$, and the bridge contains 56 cables. On the surface of the bridge model, two tracks are arranged to ensure that the track of the car is basically the same as the line at each time. In order to test the measurement accuracy of the system, the wireless measurement car is carried out on the bridge model for multiple load tests. At the same time, in order to compare with the deformation value between CDMS and leveling method, the dial indicators are mounted for recording deformation under various conditions of value in the three measuring points section (bridge span $1 / 4$, center of span and bridge span 3/4). The test scenario is shown in Fig. 4.

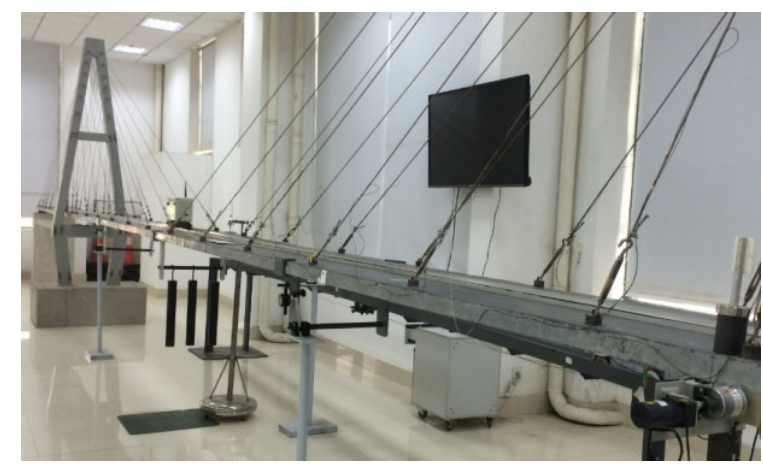

Fig. 4 Test scenario of bridge model.

\subsection{Load experiment}

At both ends of the bridge, the position sensors are installed for data calibration, and the total mileage of the car is 9.7 meters per time, respectively. Firstly, the car runs 3 times on the surface of the bridge model without external load, and by analyzing the data the repeatability of the system is shown in Fig. 5. Secondly, we simulate the load test by hanging $10 \mathrm{~kg}$ and $30 \mathrm{~kg}$ weights in the center of bridge span. The car runs 3 times in two cases repeatedly. After averaging the 3 trip data, we can get the curve of bridge structure before and after loading, as shown in Fig. 6. The horizontal axis is between the test point and the starting point of the distance, and the vertical coordinate is the bridge deck elevation for the relative starting point. The data comparison is shown in Table 1.

\begin{tabular}{lcccc}
\multicolumn{2}{l}{ Table 1 Comparison of CDMS and indicator } \\
\hline \multirow{2}{*}{$\begin{array}{c}\text { Loading } \\
\text { condition }\end{array}$} & \multirow{2}{*}{ Section position } & \multicolumn{3}{c}{ Deformation $/ \mathrm{mm}$} \\
\cline { 3 - 5 } & & CDMS & Indicator & Difference \\
\hline \multirow{3}{*}{$10 \mathrm{~kg}$} & Span 1/4 & -0.59 & -0.55 & -0.04 \\
& Center of span & -1.12 & -1.17 & 0.05 \\
& Span 3/4 & -0.36 & -0.33 & -0.03 \\
\hline \multirow{3}{*}{$30 \mathrm{~kg}$} & Span 1/4 & -1.12 & -0.19 & -0.03 \\
& Center of span & -2.70 & -2.74 & 0.04 \\
& Span 3/4 & -1.03 & -1.05 & 0.02 \\
\hline
\end{tabular}

\subsection{Summary}

From Fig. 5, the system has good repeatability and the maximum deviation is $0.08 \mathrm{~mm}$. From Fig. 6 , it can be seen that the overall alignment of the bridge after loading is moved down as compared with the no-load. The deformation trend before loading is consistent with that of loading. The bridge deformation is the maximum near the load point, and it becomes smaller and smaller with the distance from the load point. This phenomenon is consistent with the load characteristics of the structure. The continuous curve also reflects that the actual maximum deformation is not located in the center of bridge span, which is related to the adjustment of cable force of the bridge model. In addition, the maximum deviation between the CDMS and the dial indicator does not exceed $0.05 \mathrm{~mm}$, as shown in Table 1. The result shows that the CDMS can 
accurately reflect the scale bridge model loading section and the maximum deformation.

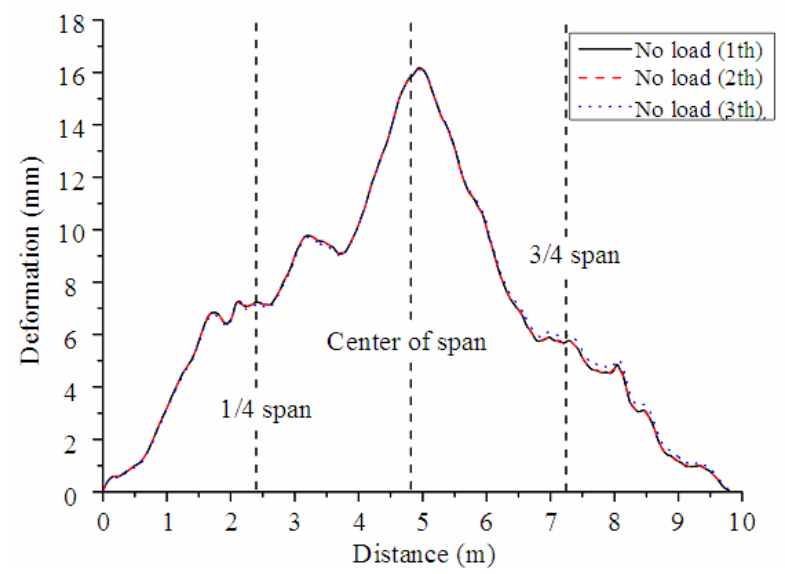

Fig. 5 Repeatability of the CDMS.

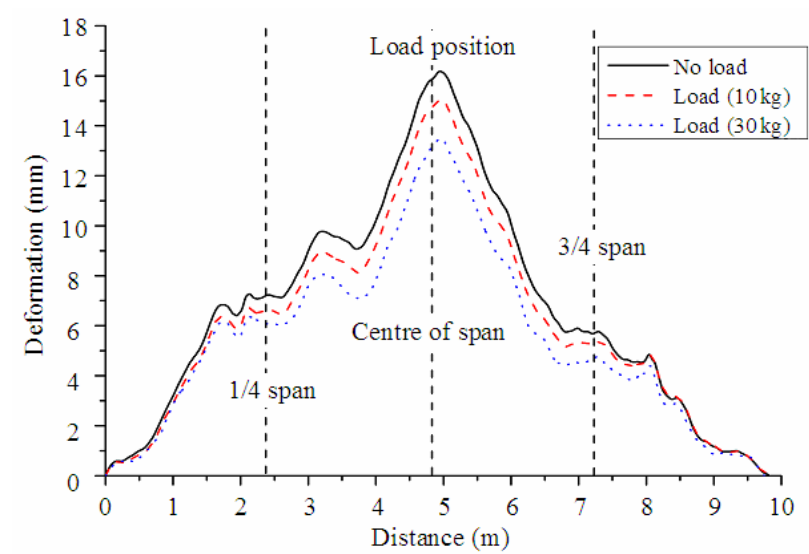

Fig. 6 Continuous curves of the bridge before and after loading.

\section{Test of real bridge}

\subsection{Engineering Survey}

A bridge across the Yangtze River is the world's longest and widest cable-stayed bridge, of which the total cost is 139 billion yuan, the length is 10 kilometers, and the width is 40.5 meters. It was designed with the standards of the eight-lane expressway, and the allowed maximum speed for vehicles is $120 \mathrm{~km} / \mathrm{h}$. The main bridge is about 2680 meters long, and it has the largest number of towers in the world. In order to ensure the safety of bridge, it is necessary to carry out the load test before the official opening. An overview of the bridge and load vehicle is shown in Fig. 7.

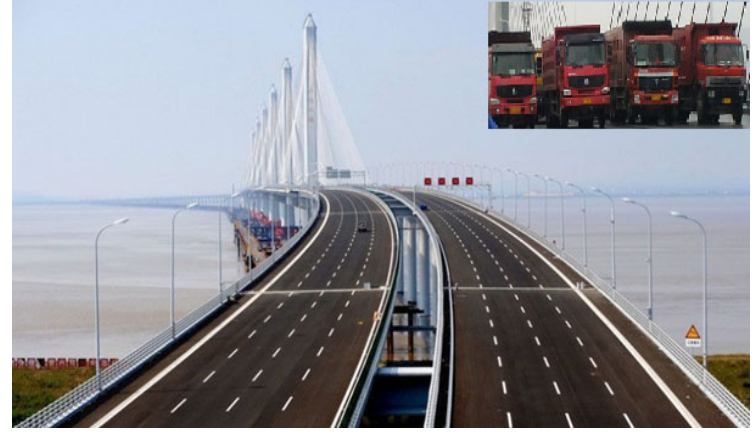

Fig. 7 Overview of a bridge across the river in China.

\subsection{Load test}

In order to further validate reliability and precision of the system in the practical application, the CDMS is used to test the continuous curve in the process of load test of a bridge, and the control points are checked by the level in partial conditions. Taking the 5\#-6\# pier loads as an example, there are 36 trains in the left and right load area of the working condition, and a train weighs $35 \mathrm{t}$. The whole process consists of 3 loading stages and 1 unloaded stage. The overall continuous curves and deformation values of the bridge are shown in Table 2 and Fig. 8.

Table 2 Z5\#-Z6\# load in the center of bridge.

\begin{tabular}{cccccc}
\hline \multirow{2}{*}{ Test position } & \multicolumn{4}{c}{ Deformation (mm) } \\
\cline { 3 - 6 } & & $\begin{array}{c}\text { First } \\
\text { stage }\end{array}$ & $\begin{array}{c}\text { Second } \\
\text { stage }\end{array}$ & $\begin{array}{c}\text { Third } \\
\text { stage }\end{array}$ & $\begin{array}{c}\text { Un- } \\
\text { load }\end{array}$ \\
\hline Z4-Z5 & Left & 0.119 & 0.133 & 0.228 & -0.023 \\
Span & Right & 0.107 & 0.175 & 0.223 & -0.026 \\
\hline Z5-Z6 & Left & -0.241 & -0.493 & -0.697 & -0.006 \\
Span & Right & -0.409 & -0.622 & -0.732 & -0.002 \\
\hline Z6-Z7 & Left & 0.089 & 0.140 & 0.227 & -0.051 \\
Span & Right & 0.103 & 0.184 & 0.260 & -0.052 \\
\hline
\end{tabular}

Note: the negative values in the table indicate the deformation of the beam.

From Fig. 8 and Table2, with an increase in the vehicle load, the deformation of the cross section is gradually increasing, and the back arch is presented in the left and right sides of the loading area. The deformation of Z4-Z5 span and Z6-Z7 span is relatively small. After unloading, the overall alignment of the bridge is basically returned to the no-load state. The phenomenon is consistent with the structural deformation characteristics of the bridge. 


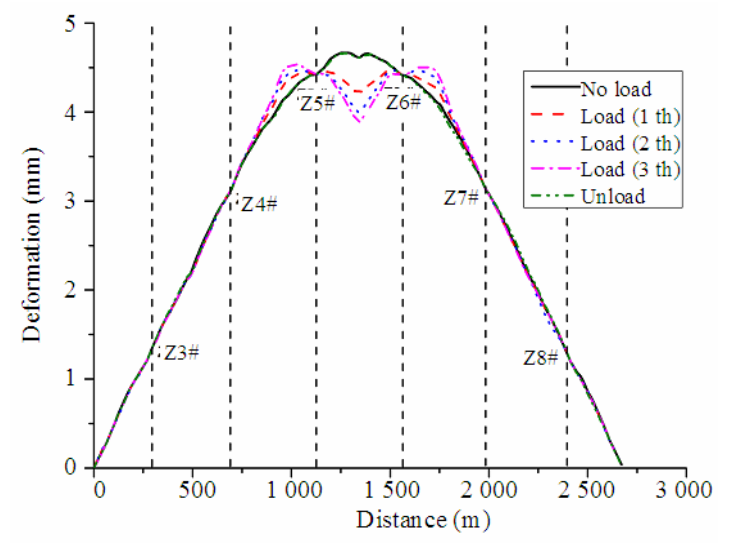

Fig. 8 Continuous curve and deformation of bridge.

At the same time, in order to test the accuracy of the system on the real bridge, the control points are checked by the level gauge in one case, and the comparison results are shown in Fig. 9 below.

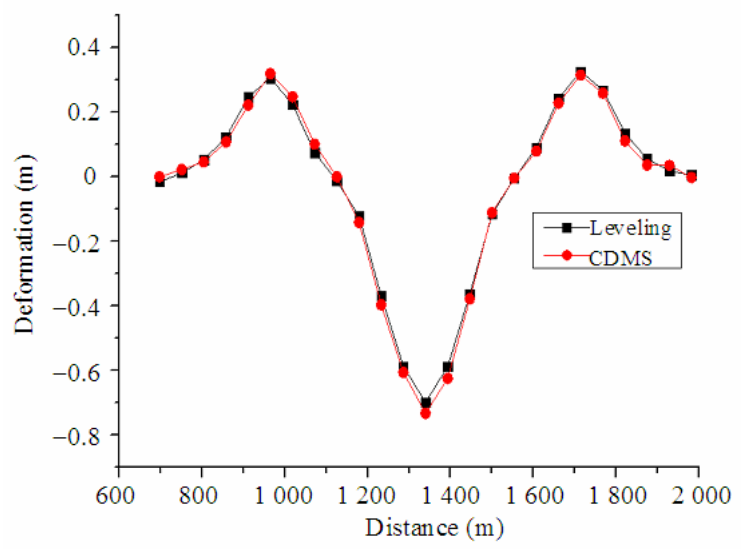

Fig. 9 Contrast curve of CDMS and level meter.

From Fig. 9, the dot represents the value of elevation obtained by the CDMS, and the square represents the value by the level meter. The measured values of the two methods are relatively close. It shows that the detection accuracy of the CDMS is almost the same as the level meter.

\subsection{Summary}

From Fig. 8, the deformation of the main girder is symmetric and consistent with the symmetric loading and the structure of the bridge. After unloading, the bridge alignment is basically recovered, which shows that the structure is of good elasticity. From Table 2, it can be known that the maximum deviation of the measured deformation is $0.0588 \mathrm{~m}$.
From Fig. 9, the maximum deviation is 0.0688 meters between the measured deformation and the curve system measured by leveling. The deviation may be caused by random errors of bridge deck pavement and test. As for the bridge, when the load deformation reaches meter level, the measurement accuracy meets the engineering requirements.

\section{Conclusions}

The system detects the surface of bridge structure in the process of carrier movement, and no sensor is needed to be installed on the structure surface. In the detection process, there is no need to seal the road, without affecting the traffic, and the system has the features of convenient, fast, continuous measurement, and high accuracy. This system can accurately locate the maximum deformation of bridge span, and it has incomparable advantages especially in the linear detection of large span bridge.

\section{Acknowledgement}

This work was supported by the National Natural Science Foundation of China (NO. 61402345), National Science and Technology Support Program (2014BAG07B01), and Fundamental Research Funds for the Central Universities Special Fund (WUT: 2014-II-012).

Open Access This article is distributed under the terms of the Creative Commons Attribution 4.0 International License (http:// creativecommons.org/licenses/by/4.0/), which permits unrestricted use, distribution, and reproduction in any medium, provided you give appropriate credit to the original author(s) and the source, provide a link to the Creative Commons license, and indicate if changes were made.

\section{References}

[1] D. Jiang, D. Sun, and L. Liang, "Slab deflection measurement technique based on fiber optic gyro," in Proc. SPIE, vol. 4077, pp. 141-144, 2000.

[2] B. F. Spencer, "Opportunities and challenges for smart sensing technology," in First International Conference on Structural Health Monitoring and Intelligent Infrastructure, Japan, pp. 65-71, 2003. 
[3] J. Ou and H. Li, "Wireless sensor information fusion for structural health monitoring," in Proc. SPIE, vol. 5099, pp. 356-362, 2003.

[4] D. V. Jáuregui, K. R. White, C. B. Woodward, and K. R. Leitch, "Noncontact photogrammetric measurement of vertical bridge deflection," Journal of Bridge Engineering, 2003, 8(4): 212-222.

[5] Y. Yu and J. Ou, "Design of wireless intelligent sensor for structural health monitoring," in IEEE Sensor Networks and Information Processing Conference, Hongkong, pp. 1-5, 2004.

[6] A. Nickitopoulou, K. Protopsalti, and S. Stiros, "Monitoring dynamic and quasi-static deformations of large flexible engineering structures with GPS: accuracy, limitations and promises," Engineering Structures, 2006, 28(10): 1471-1482.

[7] G. Liu, "Research about low frequency dynamic characteristics of deflection testing system for LianTongGuan type bridge," Dissertation for the Degree of Master of Chongqing University, China, 2007.

[8] S. Jang, H. Jo, S. Cho, K. Mechitov, J. A. Rice, S. Sim, et al., "Structural health monitoring of a cable-stayed bridge using smart sensor technology: deployment and evaluation," Smart Structures and Systems, 2010, 6(5-6): 439-459.

[9] Y. Yu, J. Ou, and H. Li, "Design, calibration and application of wireless sensors for structural global and local monitoring of civil infrastructures," Smart Structures and Systems, 2010, 6(5): 641-659.

[10] S. Li, W. Hu, Y. Yang, F. Liu, and W. Gan, "Research of FOG-based measurement technique for continuous curve modes of long span bridge," Bridge Construction, 2014, 44(5): 69-74. 